\title{
Clinical application of laparoscopic total mesorectal excision using the intersphincteric approach through the sacrococcygeal incision for treating patients with rectal cancer
}

\author{
Jian Chen ${ }^{1}$, Yan-Zhi Bo ${ }^{2}$, Feng Han ${ }^{2}$, Shu-Zhong Wang ${ }^{3}$, Kun Wu ${ }^{1}$, Jie Zhang ${ }^{1}$, Tian-Fang Xia ${ }^{1}$, Zhen-Shen Qing ${ }^{1}$, \\ Li-Qun Pang ${ }^{1}$ \\ 'Department of General Surgery, The Affiliated Huaian No. 1 People's Hospital of Nanjing Medical University, Huai'an, Jiangsu, China \\ 2Department of General Surgery, Lian'shui County People's Hospital, Lianshui, China \\ ${ }^{3}$ Department of Nuclear Medicine, The Affiliated Huaian No. 1 People's Hospital of Nanjing Medical University, Huai'an, Jiangsu, China
}

Videosurgery Miniinv 2019; 14 (2): 210-215

DOI: https://doi.org/10.5114/wiitm.2019.81316

\begin{abstract}
Introduction: Colorectal cancer is the third most common cancer causing death in Western countries; laparoscopic surgery for colorectal cancer has many advantages and thus has been used widely. Laparoscopic total mesorectal excision through the sacrococcygeal incision under direct visualization to excise distal rectal cancer is an important procedure for super-low rectal carcinomas.

Aim: To investigate the feasibility of mesorectal excision and super-low rectal carcinoma excision using the intersphincteric approach through the sacrococcygeal incision.

Material and methods: From December 2009 to June 2017, intersphincteric resection was performed through the sacrococcygeal incision; the mesentery was excised in 27 patients with rectal cancer and a contracted pelvis (the lower edge of the tumor was 4 to $7 \mathrm{~cm}$ to the anal verge) through laparoscopy in the Gastrointestinal Surgery Department of our hospital.

Results: No death was recorded during surgery. The surgical time ranged from 190 to $310 \mathrm{~min}$, the bleeding volume was 50 to $150 \mathrm{ml}$, and the post-surgical length of stay was 6 to 19 days. There were three cases of anastomotic fistulas, one case of anastomotic stenosis, and one case of fecal incontinence. Follow-up visits were scheduled for 19 patients, with a mean time of 37 months, ranging from 3 to 92 months; one case of local recurrence, one case of peritoneal metastasis, and two cases of hepatic metastasis were observed.

Conclusions: Laparoscopic total mesorectal excision using the intersphincteric approach through the sacrococcygeal incision is feasible for treating patients with a contracted pelvis and super-low rectal carcinoma.
\end{abstract}

Key words: laparoscope, rectal carcinoma, transsacral approach, intersphincteric anterior rectal resection.

\section{Introduction}

Colorectal cancer, including rectal cancer, is the third most common cause of cancer-related death in Western countries. In the treatment of colonic carcinomas, laparoscopic surgery has been proven to yield faster post-surgical recovery, fewer complications, and better cosmetic results with equal oncologic results, compared with non-laparoscopic/traditional surgery [1]. Abdominoperineal resection with permanent colostomy and sphincter-preserving (SP) resection of the rectum are the two primary and efficient surgical options for the treatment of rectal cancer $[2,3]$. Since the long-term consequences of the abovementioned

\section{Address for correspondence}

Li-Qun Pang, Department of General Surgery, The Affiliated Huaian No. 1 People's Hospital of Nanjing Medical University,

223300 Huai'an, Jiangsu, China, phone: +86 517 83165499/13515248309, fax: +86 517 83165499, e-mail: cnliqunpang@163.com 
procedures can be devastating, leading to life-altering consequences, such as permanent stoma formation [4], it is critical to cut off the tumor completely and preserve the continuity of the bowel and the function of the anal sphincter [5]. Apart from the distance of the tumor from the anal verge, other important factors, such as the patient's condition, oncologic considerations, technical feasibility, and surgeon's personal experience, were reported as risk factors that might influence the success rate of SP surgery $[6,7]$.

Nowadays, laparoscopic radical surgery of rectal cancer is widely performed [8]. Progress has been made in a domestic study of laparoscopic ultralow rectal anterior excision using the intersphincteric approach through the pelvic cavity for treating patients with rectal cancer [9].

Currently, laparoscopic total mesorectal excision (LTME) has become the gold standard technique for the surgical treatment of rectal cancer [10]. The outcomes of patients who undergo LTME are directly linked to the quality of the surgery, as it remains a challenging procedure that requires a high level of expertise, and it has a long learning curve to ensure adequate and safe resection [11].

In this study, we investigated 27 patients with low rectal cancer and a contracted pelvis from December 2009 to June 2017, in whom intersphincteric resection (ISR) was performed through a sacrococcygeal incision under direct visualization, the distal rectal cancer was excised, and anastomosis was performed. The surgical process for these patients was simplified, with qualified therapeutic efficacy. The details of the cases are reported below.

\section{Aim}

The aim of the study was to investigate the shortand long-term effects and safety of LTME and super-low rectal carcinoma excision through the sacrococcygeal incision under direct visualization.

\section{Material and methods}

\section{Clinical information}

From December 2009 to June 2017, the abovementioned procedures were performed in 27 patients: 17 men and 10 women. The mean patient age was $55.6 \pm 13.7$ years (range: $42-71$ years), and the mean course duration was 4 months (range: 3 weeks to 6 months). Enteroscopy and biopsy were performed in all patients before surgery to confirm the existence of rectal adenocarcinoma.

The mean distance between the lower edge of the tumors and the anal verge was $5.3 \pm 0.5 \mathrm{~cm}$, ranging from 4 to $7 \mathrm{~cm}$. The mean diameter of the tumors was $3.5 \pm 1.5 \mathrm{~cm}$, ranging from 1 to $5 \mathrm{~cm}$. $\mathrm{B}$-ultrasound scanning and magnetic resonance imaging (MRI) demonstrated that in all the patients, the tumor mass did not invade the anorectal ring; further, there were no enlarged para-intestinal mesenteric lymph nodes and no distal metastasis. This study was conducted in accordance with the Declaration of Helsinki. This study was conducted with approval from the Ethics Committee of the Huaian First People's Hospital. Written informed consent was obtained from all participants.

\section{Surgical procedures}

\section{Laparoscopic total mesorectal excision}

The patients were placed in the herringbone-Trendelenburg position under general anesthesia, with endotracheal intubation; artificial pneumoperitoneum was established with an intra-abdominal pressure of $12-15 \mathrm{~mm} \mathrm{Hg}(1 \mathrm{~mm} \mathrm{Hg}=0.133 \mathrm{kPa})$. Five trocars (5-10 $\mathrm{mm}$ ) were inserted into the abdomen at the place of $3.5 \mathrm{~cm}$ inward from the left and right anterior superior iliac spines, above the navel, and at the intersection of the umbilical plane and the outer edge of the right rectus abdominis and $3.5 \mathrm{~cm}$ above the pubis. The patients were examined through laparoscopy to ensure that LTME was performed only when no swollen mesenteric lymph nodes or metastasis was observed within the enterocoelia. An ultrasonic scalpel was then used in the presacral space through the sacral plane in an upward direction to separate Toldt's space and the left hemicolon. The inferior mesenteric artery was ligated at the incisal edge of the inferior mesenteric plexus plane. The inferior mesenteric vein was cut off at a high position and ligated (Photo $1 \mathrm{~A}$ ). The peritoneum outside the sigmoid colon and descending colon was incised, and the two colons were separated until the splenic flexure. Sharp dissection was performed along the loose connective tissue of the pelvic fascia between the organs and the abdominal wall until the pelvic floor (Photo $1 \mathrm{~B}$ ). Total mesorectal excision (TME) was performed along the presacral space until the area under the apex of the coccyx, with adequate protection of the 

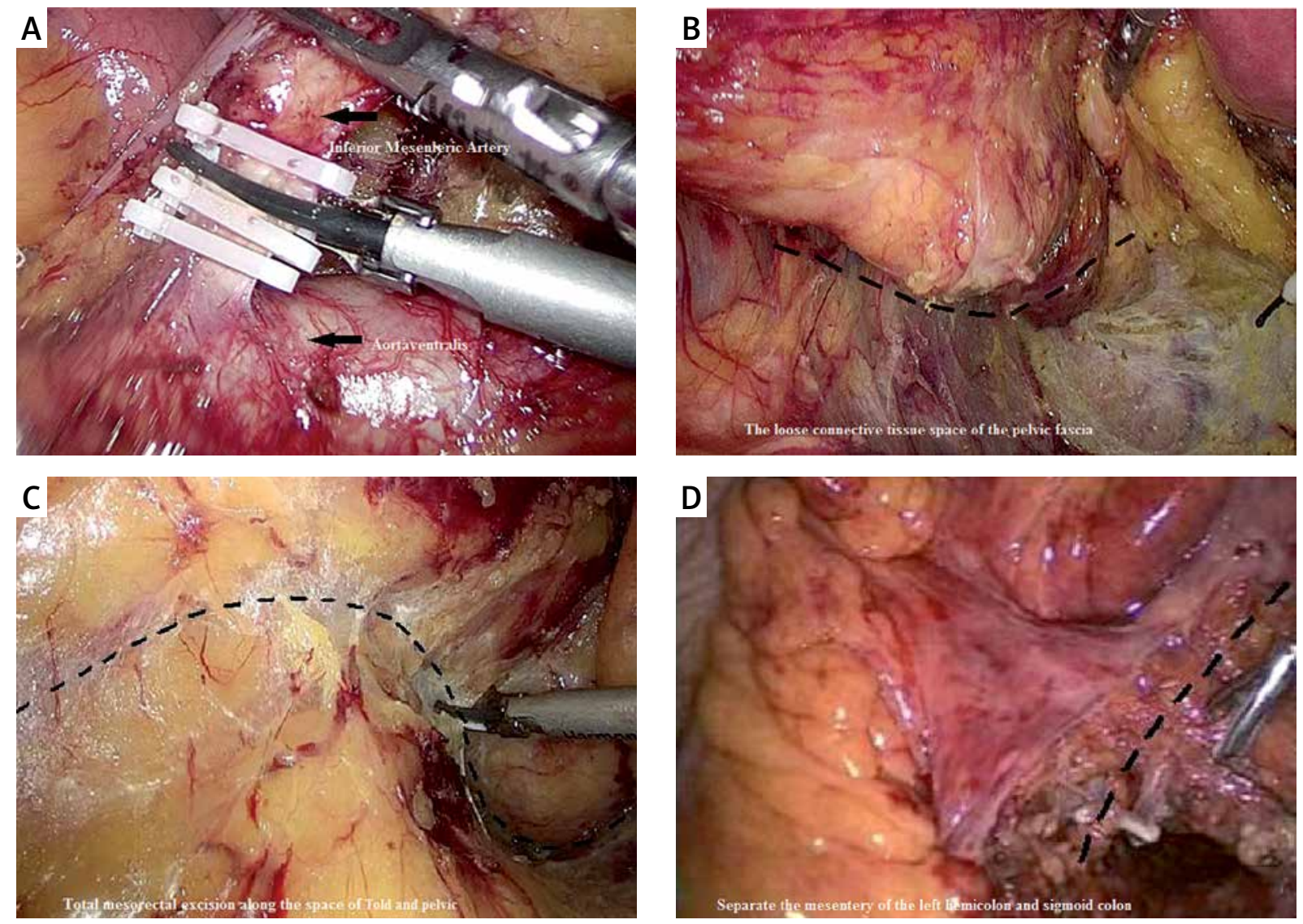

Photo 1. Mesenteric lymphadenectomy and TME. A - Resection of the inferior mesenteric artery; B - dissection along the loose connective tissue of the pelvic fascia; $\mathbf{C}$ - total mesorectal excision; $\mathbf{D}$ - separation of the mesentery of the left hemicolon and sigmoid colon

hypogastric plexus (Photo $1 \mathrm{C}$ ). The mesentery of the left hemicolon and sigmoid colon was separated until the anticipated excision plane and tagged using a titanium clip (Photo 1 D). Thereafter, the trocars were removed when no active bleeding was observed in the abdominal cavity, and the incision was subcutaneously sutured to complete the abdominal surgery.

\section{The ISR and anastomosis through the sacrococcygeal incision}

The patients were placed in the Jackknife or prone position. A $6-8-\mathrm{cm}$ midline incision was created from the upper edge of the fourth sacrum to the anal edge to cut open the skin and subcutaneous tissue and cut off the coccyx (Photo 2 A). Performing further incision of a part of the sacrum or external anal sphincter (EAS) depended on the exposed pathological changes. The patients were then divided into two groups, the deep group and the superficial group, on the basis of deep or superficial EAS incision, respectively, with suturing and tagging at the incision end for more accurate repair. The profundal fascia and the pelvic floor muscle were incised in the midline to meet the abdominal surgical plane and expose the posterior rectal wall (Photo $2 \mathrm{~B}$ ). The rectum and the sigmoid colon were lifted out from the incision to explore the locus, size, and range of the rectal carcinoma and the level involving the intestinal wall. The rectum was then separated along the pararectal space and the pelvic floor muscle from both sides toward the anterior rectal wall until fully separated. The separation was completed when it reached the plane of the dentate line in a downward manner to expose the hemorrhoids (swollen veins annularly surrounding the rectal wall). The cancerous rectum was incised more than $2 \mathrm{~cm}$ away from the distal end of the tumor under direct visualization. Closed amputation was performed at the upper and lower ends of the target rectum (Photo $2 \mathrm{C}$ ). End-to-end 

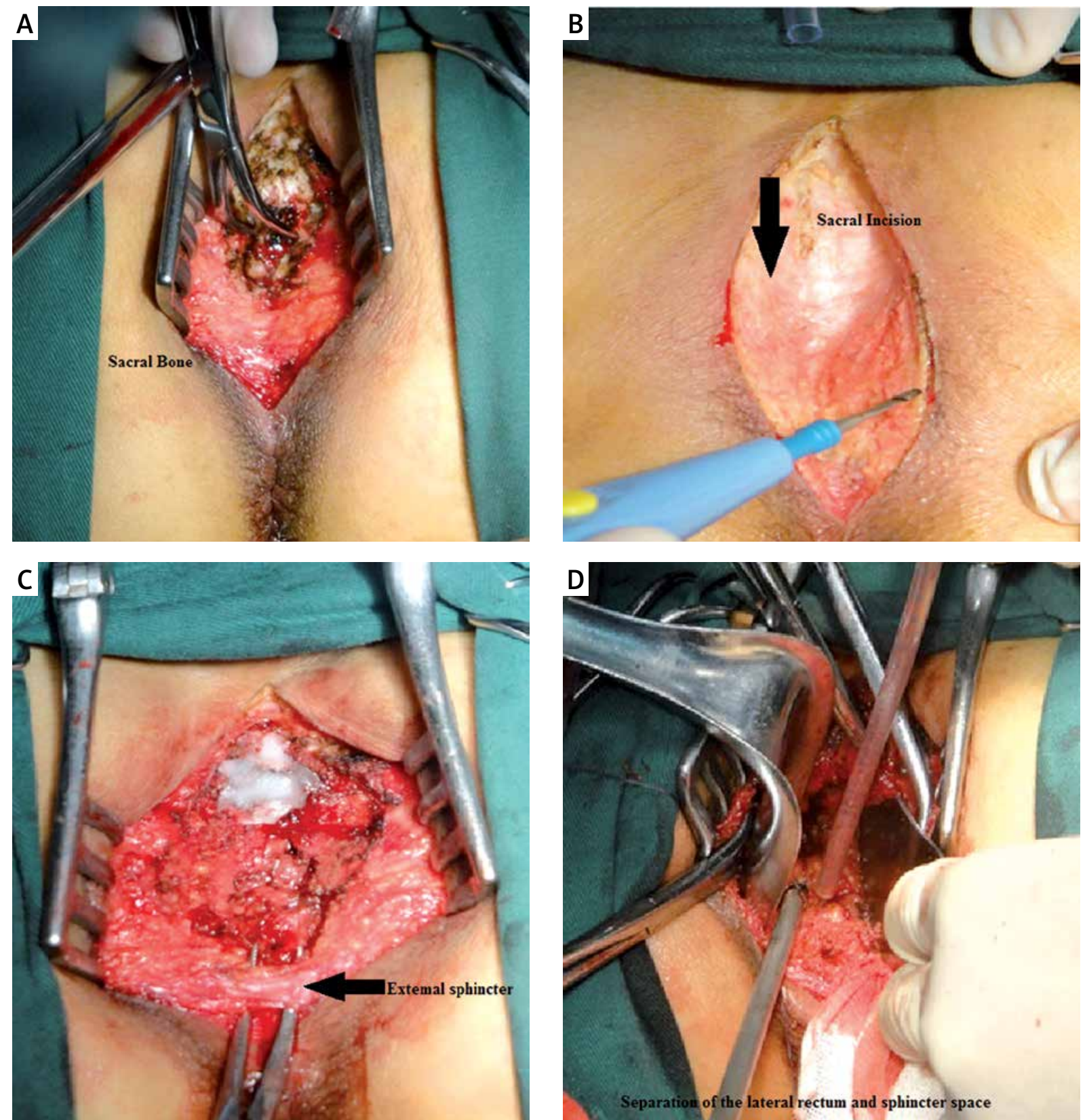

Photo 2. Sacrococcygeal surgery process. A - Incision of the upper edge of the fourth sacrum to the anal edge; $\mathbf{B}$ - cut off the external anal sphincter; $\mathbf{C}$ - the rectum was then separated along the pararectal space and the pelvic floor muscle; $\mathbf{D}$ - end-to-end anastomosis

anastomosis was completed using the dual stapler technique (Photo $2 \mathrm{D}$ ).

\section{Results}

\section{Surgical results}

All procedures were completed smoothly at a mean surgical time of 240 min, ranging from
190 to $310 \mathrm{~min}$. The mean bleeding volume was $100 \mathrm{ml}$, ranging from 50 to $150 \mathrm{ml}$. The mean distance between the anastomosis site and pectinate line was $2.9 \mathrm{~cm}$, ranging from 1.8 to $3.3 \mathrm{~cm}$. The mean post-surgical length of stay was 13 days, ranging from 6 to 19 days. Three cases of anastomotic fistula, one case of anastomotic stenosis, and one case of fecal incontinence were observed. 


\section{Pathological results}

Pathological specimens for gross examination: The distal negative part of the specimens was cut off, and the resection margin was more than $2 \mathrm{~cm}$ $(2.8 \pm 0.5 \mathrm{~cm})$. In the pathological staging, 19 cases were classified under Dukes' stage A and 8 cases under stage B. Regarding the pathological type, 8 cases were well-differentiated adenocarcinomas, 14 cases were moderately differentiated adenocarcinomas, and 5 cases were poorly differentiated adenocarcinomas. Follow-up visits were scheduled for 19 patients, with a mean duration of 37 months, ranging from 3 to 92 months, during which local recurrence was observed in 1 case, peritoneal metastasis in one case, and hepatic metastasis in 2 cases.

\section{Discussion}

As an improved technique, laparoscopy significantly enhances the surgical skills of surgeons and the surgical efficacy of low rectal cancer resection and results in a shorter recovery time after surgery, lower occurrence rate of complications [12], and more reliable long-term results of radical surgery [13] than those for traditional surgical techniques. Previous studies have demonstrated many cases of laparoscopic low anterior resection or low anterior resection using a prolapsing technique with a certain curative effect [14-16]. The long-term oncological and functional outcomes of patients who underwent LTME were good [17], including those of patients with obesity and the elderly $[18,19]$.

However, it is usually difficult to achieve radical cure and preserve the sphincter simultaneously in TME during ultra-low rectal carcinoma resection, in which case the former is prioritized. Conversely, sufficient attention should be paid to strict control of surgical indications and non-tumor technology, besides radical cure and sphincter preservation [20, 21].

The major indication for rectal carcinoma resection leading to poor radical cure and surgical failure remains local recurrence of carcinoma. Many factors can result in recurrence; among these, insufficient resection range or residual tumor cells at the resection margin, but not residual tumor cells in the lateral rectal lymph node, are indicated to be the main factors [22].

The rectum below the levator hiatus surface is not surrounded by the mesentery but is surrounded annularly by the puborectalis and deep part of the EAS muscle. During ISR, performed as the SP proce- dure for ultra-low rectal carcinoma, these muscles should be separated from the longitudinal rectal muscle via sharp dissection. In some special cases, such as cases with a contracted pelvis, obesity, or anterior rectal wall carcinoma, the ultra-low rectum within the rectal mesentery and sphincters cannot be precisely exposed and completely separated through laparoscopy. Improper surgical procedure and excessive extrusion or traction, for instance, may lead to loss of the integrity of the rectal wall and mesentery and further shedding and diffusing of tumor cells or residual tumor cells at the distal resection margin, which finally affect the radical cure outcomes of the surgery.

In clinical cases in which the target was difficult to expose through laparoscopy owing to the presence of a contracted pelvis, an incision was created within the sacrococcygeal area based on Mason's operation. In entering the pelvic floor fascia, the surgical passageway can then meet the intra-abdominal surgical plane above the coccyx plane, which is easier and more reliable than the approach in Mason's operation. Such a wide surgical horizon leads to exquisite anatomical separation of the rectum within the sphincters under direct visualization, yielding more accurate surgical procedures; this reduces the possibility of injury to the prostate or posterior virginal wall, ensures the range for carcinoma resection, and improves the quality of coloanal anastomosis, finally yielding a safer and more reliable operation. The resection range and negative resection margin were guaranteed in all cases in the study.

The complications of SP LTME using the intersphincteric approach through the sacrococcygeal incision were similar to those of laparotomy, Mason's operation, and laparoscopic surgery, including wound infection, anastomotic fistula, and fecal incontinence. Experience in performing laparoscopy, laparotomy, and Mason's operation is required for doctors performing the surgery, who can properly cut and repair the EAS to avoid anal incontinence. Moreover, performing the surgery under direct visualization can ensure the quality of anastomosis and reduce the occurrence rate of anastomotic fistulas. Local wound infection was a common complication after surgery, mostly resulting from the fluid that accumulated in the presacral space, as the local defect was difficult to suture and the recovery time was usually long after the resection of the coccyx. Therefore, adequate local drainage was critical for 
preventing wound infection. The infection observed in this study was caused by the fluid in the presacral space after surgery.

Although excision through the sacrococcygeal area can ensure the quality of local resection, proper evaluation before surgery is key to a radical cure. If early-stage carcinoma is indicated in the pre-surgical evaluation and is found to be in the progressive stage at the post-surgical evaluation, additional radiotherapy is necessary to reduce the recurrence rate of the carcinoma.

\section{Conclusions}

The LTME using the intersphincteric approach through the sacrococcygeal incision is effective and safe for patients with contracted pelvis and super-low rectal carcinoma; it yields shorter hospital length of stay and better short- and long-term outcomes.

\section{Acknowledgments}

Jian Chen and Yan-Zhi Bo contributed equally to this work and should be considered co-first authors.

\section{Conflict of interest}

The authors declare no conflict of interest.

\section{References}

1. Vennix S, Pelzers L, Bouvy N, et al. Laparoscopic versus open total mesorectal excision for rectal cancer. Cochrane Database Syst Rev 2014; 4: CD005200.

2. Mak JCK, Foo DCC, Wei R, Law WL. Sphincter-preserving surgery for low rectal cancers: incidence and risk factors for permanent stoma. World J Surg 2017; 41: 2912-22.

3. Cong ZJ, Hu LH, Xing JJ, e al. Risk factors associated with sphincter-preserving resection in patients with low rectal cancer. Int Surg 2014; 99: 330-7.

4. Yeo HL, Abelson JS, Mao J, et al. Minimally invasive surgery and sphincter preservation in rectal cancer. I Surg Res 2016; 202: 299-307.

5. Zhu HB, Wang L, Li ZY, et al. Sphincter-preserving surgery for low-middle rectal cancer: can we predict feasibility with high-resolution magnetic resonance imaging? Medicine 2017; 96: e7418.

6. Paquette IM, Kemp JA, Finlayson SR. Patient and hospital factors associated with use of sphincter-sparing surgery for rectal cancer. Dis Colon Rectum 2010; 53: 115-20.

7. Zhou X, Wang B, Li F, et al. Risk factors associated with nonclosure of defunctioning stomas after sphincter-preserving low anterior resection of rectal cancer: a meta-analysis. Dis Colon Rectum 2017; 60: 544-54.
8. Hisada M, Katsumata K, Ishizaki T, et al. Complete laparoscopic resection of the rectum using natural orifice specimen extraction. World J Gastroenterol 2014; 20: 16707-13.

9. Klose J, Tarantino I, Kulu Y, et al. Sphincter-preserving surgery for low rectal cancer: do we overshoot the mark? J Gastrointest Surg 2017; 21: 885-91.

10. Young M, Pigazzi A. Total mesorectal excision: open, laparoscopic or robotic. Recent Results Cancer Res 2014; 203: 47-55.

11. Chang TC, Kiu KT. Transanal total mesorectal excision in lower rectal cancer: comparison of short-term outcomes with conventional laparoscopic total mesorectal excision. J Laparoendosc Adv Surg Tech A 2018; 28: 365-9.

12. Bonjer HJ, Hop WC, Nelson H, et al. Laparoscopically assisted vs open colectomy for colon cancer: a meta-analysis. Arch Surg 2007; 142: 298-303.

13. Lezoche G, Baldarelli M, Campagnacci R, et al. Laparoscopic surgery of rectal cancer: state of art and long-term results. Ann Ital Chir 2010; 81: 265-8.

14. Juul T, Ahlberg M, Biondo S, et al. Low anterior resection syndrome and quality of life: an international multicenter study. Dis Colon Rectum 2014; 57: 585-91.

15. Fukunaga M, Kidokoro A, Iba T, et al. Laparoscopy-assisted low anterior resection with a prolapsing technique for low rectal cancer. Surg Today 2005; 35: 598-602.

16. Cong JC, Chen CS, Ma MX, et al. Laparoscopic intersphincteric resection for low rectal cancer: comparison of stapled and manual coloanal anastomosis. Colorectal Dis 2014; 16: 353-8.

17. Bakker IS, Snijders HS, Wouters MW, et al. High complication rate after low anterior resection for mid and high rectal cancer; results of a population-based study. Eur J Surg Oncol 2014; 40: 692-8.

18. Levic K, Bulut O, Schødt M, Bisgaard T. Increased perirenal fat area is not associated with adverse outcomes after laparoscopic total mesorectal excision for rectal cancer. Langenbecks Arch Surg 2017; 402: 1205-11.

19. Lakkis Z, Panis Y. Is there any reason not to perform standard laparoscopic total mesorectal excision? Clin Colon Rectal Surg 2017; 30: 333-8.

20. Nelson H, Petrelli N, Carlin A, et al. Guidelines 2000 for colon and rectal cancer surgery. J Natl Cancer Inst 2001; 93: 583-96.

21. Syk E, Torkzad MR, Blomqvist L, et al. Radiological findings do not support lateral residual tumour as a major cause of local recurrence of rectal cancer. Br J Surg 2006; 93: 113-9.

22. Akagi Y, Kinugasa T, Shirouzu K. Intersphincteric resection for very low rectal cancer: a systematic review. Surg Today 2013; 43: 838-47.

Received: 15.07.2018, accepted: 30.10.2018 